\title{
A Survey of Short-term Memory in Consecutive Interpreting Course
}

\author{
Leina $\mathrm{Lu}$ \\ school of Foreign Languages, school of Humanities and \\ Social Science \\ Nanjing University of Science and Technology \\ Nanjing, China \\ luleina@mail.njust.edu.cn
}

\author{
Ye Chen \\ school. of Foreign Languages \\ Nanjing University of Science and Technology \\ Nanjing, China
}

\begin{abstract}
Interpreting is a complicated procedure of information processing relating to people's logic and psychological process. Memory is the key link and the major obstacle affecting interpreting. Interpreting is an instant logic conversion activity between two languages, conveying the information in target language on a basis of accurate understanding in a short period of time, which requires interpreters to maintain and process information which highly depends on short-term memory. This paper endeavors to analyze the status and functions of short-term memory objectively through questionnaire sent to English majors about the opinions of the relationship between short-term memory and interpreting quality.
\end{abstract}

Keywords-consecutive interpreting; short-term memory; memory training

\section{INTRODUCTION}

Interpreting is a real-time oral translation activity based on language information. From the perspective of psychology, interpreting is psychological and logical information activity relating to different languages. The nature of interpreting is interpreter's memory process and semantic transformation of different languages, requiring short-term retention of words and their meanings in the source and target languages, while simultaneously processing this information for understanding, translation, and production (Cowan, 2000; Daro, 1994). Interpreter is required to acknowledge and retain the information while listening and re acknowledge and memorize while interpreting. Thus, memory in interpreting is actually interpreter's retention and extraction of processed and encoded information.

A good memory is a necessary requirement for interpreters, which affecting the quality of interpreting directly. A complete memory system is supposed to include sensory memory, short-term memory and long-term memory. Due to the nature of interpreting, short-term memory enjoys an important role in interpreting. Since college interpreting course mainly focuses on consecutive interpreting, this paper endeavors to analyze the status and functions of short-term memory in consecutive interpreting objectively.

\section{LITERATURE REVIEW}

A complete memory system is supposed to include sensory memory, short-term memory and long-term memory. Sensory memory is the first tunnel that all the information enters the brain, a sensory retention of brain's reflection to external stimuli, which only lasts $0.25 \sim 2$ seconds and mostly ends up being forgotten while some information is restored due to attention and enters shortterm memory. Information will be encoded when turns into short-term memory from sensory memory in acoustic or linguistic form but the retention time is very short. Shortterm memory is a processing device or filter before information entering cortex and becoming long-term memory. Long-term memory is the major instrument that brain stores information with a larger span and longer retention time from 1 minute to the whole life-time.

Miller (1956) pointed out that short-term memory's span is $7 \pm 2$ chunks and due to individual difference the amount of message is around 5 to 9 . As a special language processing activity, interpreting gives more prominence to instantaneity and extemporaneousness. Different from written translation, interpreters are not given enough opportunities to inculcate the messages and listen to them at one time. With the help of note-taking, interpreters are supposed to finish a series of frequently coinstantaneous processing tasks involving message input, temporary memory storage, organizing, expressing and monitoring. Short-term memory plays an important role in the message storage and processing.

It requires interpreter to find out the equivalent words to any numbers, terminologies, abbreviations and proper nouns in a few seconds and speak out rapidly, otherwise, these messages will simply disappear in short-term memory. The limitation of short-term memory impedes interpreters' memory. Many other factors have influence interpreter's memory, such as the speaker's speed, accent, language features and the amount of messages per unit time; the content's degree of difficulty, the nature of the task, etc. Certainly, interpreter's personal qualifications, such as the ability of listening, comprehension, memorization, notetaking, preparation, psychological diathesisand physical condition, also matters.

Since the research on interpretation officially began in 1960s, the relationship between memory ability and 
interpreting has been the one of the focus subjects (Gile, 2000). Especially after 1990s, under the influence of cognitive psychology and psycholinguistics, it is at the core of the cognitive study of interpreting (Liu Heping, 2005: 2426; Danks, et al., 1997; Moser-Mercer, 2000; Christoffels \& De Groot, 2005). However, from the perspective of research methodology, most former studies investigate and analyze the relationship by controlling variables in experimental scenarios using cognitive psychology and psycholinguistics measuring instruments. On one hand, this empirical experimental research can draw a statistical conclusion through quantitative description and analysis to the research subjects. On the other hand, the representativeness are frequently under different levels of influence of the research subjects different performance from casual occasions because of the strict variable control (Chen Jianlin, 2004: 74-77).

Western and eastern researchers notice and emphasize on memory's role in interpretation (Zhang Wei, 2006: 6670). Studies on interpreting memory show a new trend which is experiment-oriented (Gile, 2000; PE chhacker, 2004). In consecutive interpreting, the pressure of instant interpreting is relatively lower and with the help of note the short-term memory burden can be largely reduced (Zhang Wei, 2006: 66-70). Thus, note-taking becomes an important subject and the interactive relationship between memory and note is of research significance. However, due to the interactive influence of memory and note on consecutive interpretation's quality, people's recognition of memory's effect on consecutive interpretation is still based on experiences and not much data can objectively provide evidence to show the real relationship between memory ability and consecutive interpreting. So this paper intends to find out what is the role memory ability plays in consecutive memory through research and literature review.

\section{RESEARCH METHODOLOGY}

\section{A. Description of Subjects}

The respondents were English majors from Nanjing University of Science and Technology who had interpreting course. 62 participants were female students and 21 were male, among which 79 were seniors and 4 were juniors. All the participants attended interpreting course for two semesters.

\section{B. Research Instrument - Questionnaire}

This questionnaire was designed to acquire a general understanding of how much English majors know about interpreting, the common difficulties occurring in daily interpreting, opinions about memory training, the differences between English to Chinese and Chinese to English interpreting, and whether memory strategies work.

The questionnaire was given to respondent on March $26^{\text {th }}$. The author first clarified the purpose and gave explanation of each question, then asked the respondents to finish the questionnaire on the spot and collected the questionnaires. 86 copies of questionnaires were distributed. The response rate was $100 \%$ and 82 questionnaires were available. Then, the available questionnaires were classified and counted and the data were transferred to percentage of the total number. Then, the final data and charts were generated using Excel and SPSS v19.0.

\section{RESULTS AND DATA ANALYSIS}

\section{A. Respondents' General Understanding of English Interpreting}

To know how well the respondents know about English interpreting and opinions of interpreting course helps to acquire a general understanding of the respondents attitude and knowledge of English interpreting.

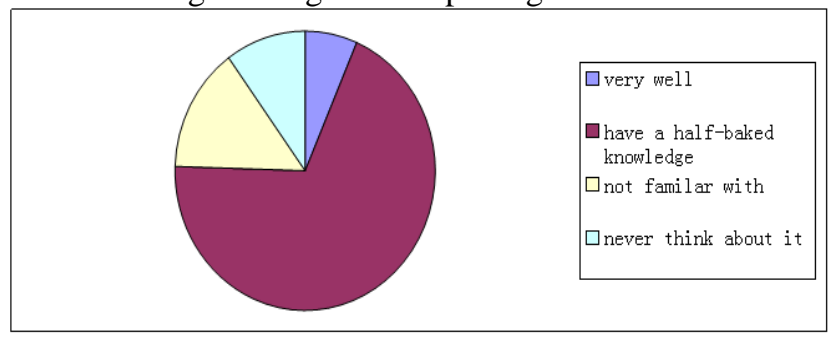

Figure 1. Chart 1. How well do you know about English interpreting?

Chart 1 shows that $6.5 \%$ of respondents chose "very well", 69\% "have a half-baked knowledge", and 14.5\% "not familiar with". It can be seen that most of the respondents have basic knowledge of English Interpreting.

Chart 2 presents students expectation of content be taught in interpreting course, which offers advice for teachers' preparation for this course.

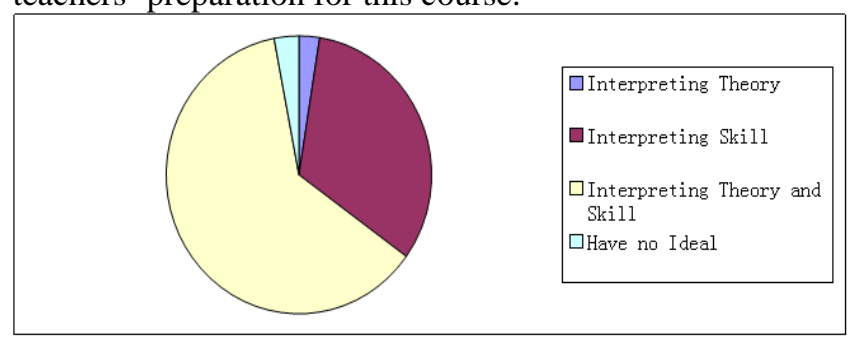

Figure 2. Chart 2. What should be taught in interpreting course?

$2.5 \%$ of respondents' answer were "Interpreting Theory", $32.5 \%$ were "Interpreting Skill", 62\% were "Interpreting Theory and Skill" and 3\% "Have no Ideal". This answer reflected what the respondents were expected to learn in the class. And it implies that the students pay attention to not only practical skill training, but also theoretical knowledge. Interpreting course should involves both skill and theory to suit the students' need for knowledge.

\section{B. Difficulty}

In real-time interpreting, apart from memory ability, many other elements have influence on the quality of memory. Among these factors, language base, interpreting skills, cognitive memory ability are considered as core factors affecting the quality of interpreting while the others are classified as external influence (PÊchhacker 2004). The 
author intended to find out what the obstacle is in interpreting.

\begin{tabular}{|l|} 
WWeak Base \\
QPoor Management of \\
Interpreting Skills \\
QProne to be Nervous \\
Oncomprehensive \\
Encyclopedic Knowledge \\
Fail to Memeorize \\
QPoor Listening
\end{tabular}

Figure 3. What impedes your interpreting performance most?

$12.5 \%$ of respondents referred to "Weak Base" as the greatest difficulty in interpreting, 21.5\% "Poor Management of Interpreting Skills", 7.5\% "Prone to be Nervous", $11.5 \%$ "Incomprehensive Encyclopedic Knowledge", 39\% "Fail to Memorize" and 8\% "Poor Listening".

According to Peterson's (1968) short-term forgetting curve, when delay time is 3 seconds, the testees' average accuracy of memory amounts to $80 \%$, but with the lengthening of delay time, the accuracy drops sharply. When delay time is 18 seconds, the accuracy is only around $10 \%$. In consecutive interpreting, delay time frequently is over several minutes, sometimes even over 15 . There is no wonder why most students chose "Fail to Memorize".

Besides, a rather large number of respondents chose "Poor Management of Interpreting Skills". Interpreting skills mainly refer to strategies and skills of language switching, including reasonable judgment of interpreting information and effective application of various interpreting strategies. Meanwhile, under the same conditions, the higher interpreting skills are, the more proficient the application is, the better the interpreting quality is (Gile, 2009).

$12.5 \%$ of respondents chose "Weak Base" and $8 \%$ chose "Poor Listening". Interpreting process is divided into 3 stages: listening-understanding-expressing and understanding is the most important link. Through listening comprehension, linguistic notations are understood and analyzed (Danica Seleskovitch, 1992). Weak base sets limitation to find exact equivalent to express.

As the respondents are not professional interpreters, lack of interpreting experience make some students feel under great pressure. Although during interpreting, memory storage mainly depends on short-term memory, the coordination of short-term and long-term memory is indispensable to memory quality on the whole. Long-term memory is the solid ground for short-term memory because comprehension of interpreting information requires a combination of rich reserve of knowledge and short-term memory. If accumulated information can be used and helps to process new information, the efficiency of short-term memory will be enhanced (Liu Jing, 2010). As a result, interpreters should acquire a wide range of knowledge, broaden the horizon and diversify experience to enrich the contents of long-term memory and optimize storage structure.

\section{Opinions on Cognitive Ability's Status in Interpreter's Basic Qualifications}

Cognitive ability includes memory ability and reactive ability, etc (Bao Gang, 1998). To know the role of cognitive ability from student's point of view helps to understand the importance of cognitive ability.

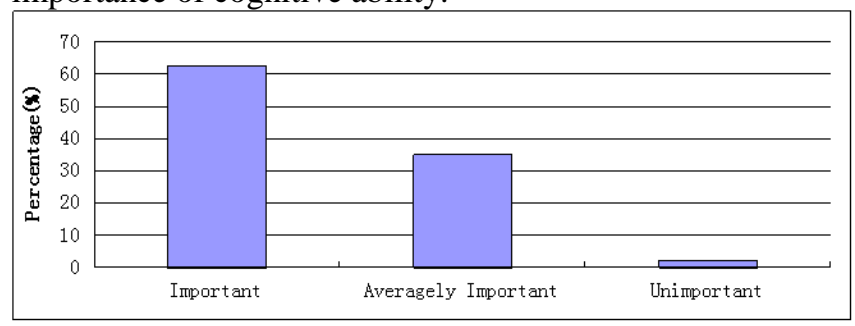

Figure 4. How do you value cognitive ability's status in interpreter's basic qualifications?

$62.5 \%$ of participants regarded cognitive ability as important, $35 \%$ averagely important and $2.5 \%$ not important. In Zhang Wei's research (2006), professional interpreters tended to regard cognitive ability's rank as averagely important while student interpreters who already begin interpreting training but not officially receive simultaneous interpreting training considered cognitive ability's rank as very important. Zhang concluded that with the improvement of interpreting practices, the influence of cognitive ability will be reduced. Thus, the students should obtain more interpreting practices to accumulate experience.

\section{Influence of Special Memory Training on Interpreting Quality}

To know whether memory training works offers teachers suggestions whether to include memory training in class organization.

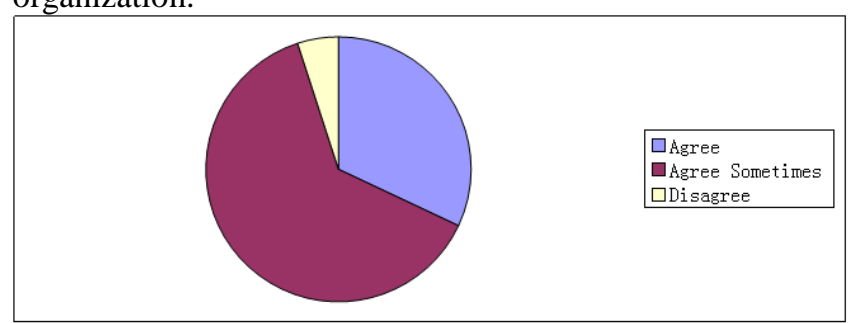

Figure 5. Do you agree that memory training improve interpreting performance?

$63 \%$ of participants chose "Agree Sometimes", 32\% "Agree" and 5\% "Disagree". Most of participants chose "Agree Sometimes" reflected that they were not sure that whether special memory training is propitious to interpreting performance improvement. However, in the meanwhile, the percentage of participants who chose "Agree" was clearly higher than those who chose "Disagree" reflected that although a majority of participants were not sure about the affect of memory training, most of them tended to admit that memory training is propitious to interpreting. To include memory training may do good to enhance memory ability. 


\section{E. Memory's Working Form in Interpreting}

First, cognitive psychology testifies that short-term memory working ability refers to not only the memory span that a task requires (Daneman, M. and P. A. Carpenter, 1980), but also efficiency of short-term memory as a resource, that is, limited memory resources being allocated properly(Daro, V., 1995: 450-466). Thus, memory span and coordination actually support and affect each other.

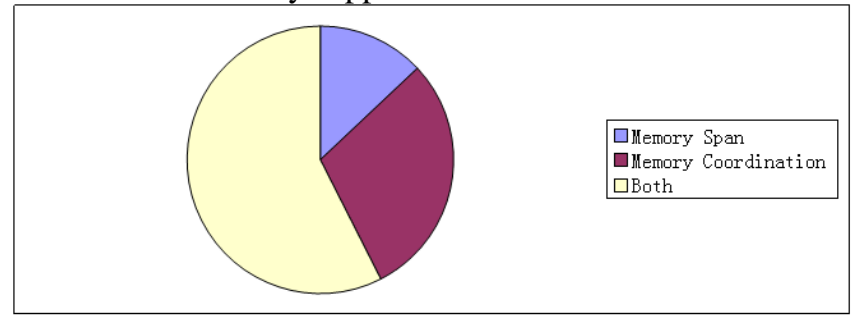

Figure 6. In which form memory works in interpreting?

$13 \%$ of participants chose "Memory span", $22.5 \%$ "Memory Coordination" and 57.5\% "Both". On the whole, the number of participants choosing "Memory span" is the lowest and the number choosing "Both" is the highest which claims that memory span solely is not the most significant factor affecting interpreting performance while memory coordination ability, especially the comprehensive ability of memory span and coordination is of great significance. Thus it is suggested that more practices and different kinds of texts will help student to understand how to assign memory span in different tasks and which part deserves better attention.

To sum up, students pay attention to both interpreting skill and theory. Then, student interpreters have problems mainly in memory span and interpreting skills. Student interpreters tend to regard cognitive ability is of great importance while professional interpreters think that cognitive ability is not very important and its function is less than language knowledge and interpreting skill, etc. Besides, although it is not quite sure that whether special memory training is useful, it is generally believed that interpreting training and practices can promote memory ability's development. It seems that skill of memory coordination is of great importance.

It is suggested that interpreting course should include interpreting skill and theory. External influence is as important as memory ability itself and in order to overcome the limitation of short-term memory, special memory training and offering more opportunities to practice interpreting are highly recommended for student interpreter to prepare themselves for different kinds of tasks. Memory span alone is not the major factor affecting interpreting quality and cooperation of memory coordination and span has greater influence on interpreting. Thus, how to reasonably assign interpreter attention to each part of the text should be taught and practiced.

Students should try to acquire a wide range of knowledge, broaden the horizon and diversify experience to enrich the contents of long-term memory and optimize storage structure. Moreover, students are supposed to practice as much as possible to accumulate more experience to cope with different situations. Students can try to encode consciously what they heard in order to make context more meaningful and easier to memorize.

\section{ACKNOWLEDGMENT}

This paper was supported by "the Fundamental Research Funds for the Central Universities", No. 30920130132036; Philosophy and Social Science Fund of Jiangsu Provincial Education Department: Studies on Short-term Memory Training in Consecutive Interpretation-a Psychological Perspective, No. 2013SJD740016.

\section{REFERENCES}

[1] Christoffels, I. K. And De Groot, A. M. B. Simultaneous Interpreting: A cognitive perspective. Oxford: Oxford University Press, 2005.

[2] Cowan, N. Processing limits of selective attention and working memory: Potential implications for interpreting. Interpreting, 2001(5), pp.117-46.

[3] Dank, J. H. , Shreve, G. M. , Fountain, S. B. , and McBeath, M. K. . Cognitive Process in Translation and Interpreting. London: SAGE Publications, 1997.

[4] Daneman, M. and P. A. Carpenter. Individual Differences in Working Memory and Reading. Journal of Verbal Learning and Verbal Behavior, 1980(19), pp.450-466

[5] Daro, V. Non-linguistic factors influencing simultaneous interpretation. S. Lambert \& B Moser-Mercer(eds), Empirical research in simultaneous interpretation. Philadelphia:John Benjamins, 1995.

[6] Gile, Daniel. Issues in Inter Disciplinary Research into Conference Interpreting: Language Processing and Simultaneous Interpreting. Amsterdam: John Benjamins Publishing Company, 2000.

[7] Gile, Daniel. Basic Concepts and Models for Interpreter and Translator Training. Amsterdam: John Benjamins Publishing Company, 2009.

[8] Miller, A. George. The Magic Number Seven Plus or MinusTwo: Some Limits on Our span for Processing Information. The Psychological Review, 1956(2), pp.81-97.

[9] Moser-Mercer, B.. Simultaneous interpreting: Cognitive potential and limitations. Interpreting, 2000.

[10] PÊ chhacker, F.. Introducing Interpreting Studies. London: Routledge Publishing Company, 2004.

[11] Peterson, L. R. \& Peterson, M. T. Short-term retention of individual verbal items. Journal of Experimental Psychology, 1968.

[12] Bao Gang. Introducing Interpreting Studies. Beijing Tourism Education Press, 1998.

[13] Chen Jianlin. Theory and Methodology in Modern Foreign Language Teaching and Research. Shanghai Foreign Language Education Press, 2004.

[14] Danica Seleskovitch. Interpreter Skills: Experience on Consecutive Interpretation and Simultaneous Interpretation. Translated by Huang Weiyi, Qian Huijie. Shanghai Translation Publishing Press, 1992.

[15] Liu Heping. An Analysis of the Achievements and Trends of Interpretation Theories. Chinese Translators Journal, 2005(4), pp.2426.

[16] Liu Jing. On Interpretation Memory and its Improving Strategies. Journal of Huaiyin Institute of Technology, 2010(19), pp. 26-29.

[17] Zhang Wei. Interpretation and Memory: History, Present and Future. Foreign Languages Research. 2006(6), pp. 67-70. 